\title{
Topology Optimization of Adaptive Compliant Aircraft Wing Leading Edge
}

\author{
M.J. Santer* and S. Pellegrino ${ }^{\dagger}$ \\ University of Cambridge, Cambridge, CB2 1PZ, UK
}

\begin{abstract}
A network analysis technique is introduced which may be used for determining and parameterizing the load paths in a lattice structure to enable a load path-based topology optimization to be carried out in a way that permits the use of Genetic Algorithms. This technique provides the designer with a greater level of control over the problem complexity than alternative schemes and enables the use of complex starting topologies. The optimization process is implemented in commercially-available software - Samcef and Boss-quattro - and a complete design process of an adaptive compliant aircraft wing rib from conception via optimization to fabrication of a demonstration model is illustrated.
\end{abstract}

\section{Introduction}

Compliant structures replace the functionality of conventional mechanism elements, for example hinges and sliders, with flexing elastic components. ${ }^{1}$ They offer a number of advantages over conventional mechanisms, including increased simplicity, reduction in number of parts, and reduced maintenance requirements. This makes them attractive for a number of applications.

The application that forms the basis of the analysis presented in this paper is the concept of a compliant structure forming the adaptive leading edge of an aircraft wing rib, suggested by Kota and co-workers. ${ }^{2}$ In this concept, a change in aerodynamic performance of a wing is obtained by morphing the cross-section of the wing itself, rather than by means of flaps. This approach can result in substantial improvements in the wing's aerodynamic efficiency. ${ }^{2}$ The wing morphing is achieved by replacing the leading edge section of each rib with an optimized compliant structure which, in response to a single displacement actuation, elastically deforms in conjunction with the wing skin resulting in a shape-change that changes the aerodynamic performance of the wing. An illustration of this concept is shown in Fig. 1 in which the leading edge has a length equal to a quarter of the wing chord length.

The design of a compliant rib leading edge is a complex process as a large number of design variables are involved. The effects of aerodynamic loading on the deformation of the compliant structure, the coupling of the compliant rib with the wing skin, and the geometric nonlinearity associated with large displacements are some of the issues that must all be addressed. Optimum solutions may not be intuitive structures as the result of a large number of design variables is that the design space is not well understood. As such, the problem is well-suited to computational optimization.

The requirement is therefore to develop a method which can synthesize and optimize both the topology and geometry of compliant structures. In order to be able to account for nonlinear behaviour and multiple forms of loading, commercially available finite element analysis software - Samcef ${ }^{3}$ - is used. Samcef is well integrated with the Boss-quattro ${ }^{4}$ software package which controls the analysis of parameterized models and contains implementations of a number of optimization algorithms.

This paper is arranged as follows. After this introduction, Section II provides a general introduction to the optimization problem definition. Sections III and IV introduce a technique to ensure that the structures generated by the optimization algorithm are not only adequately parameterized but are also physically feasible.

Using the chosen structural parameterization scheme, it is noted that in all but the most simple cases the required number of parameters to capture all possible topologies becomes prohibitively large. It is shown

*Research Associate, Department of Engineering, Trumpington Street, Member AIAA.

${ }^{\dagger}$ Professor of Structural Engineering, Department of Engineering, Trumpington Street, Associate Fellow AIAA. 


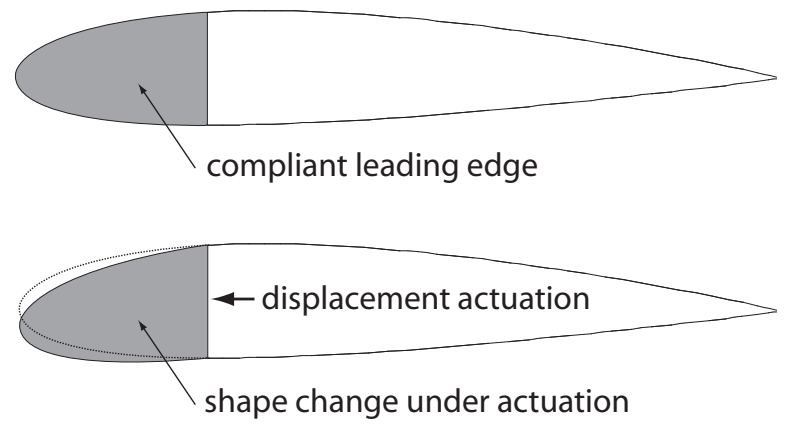

Figure 1. Compliant adaptive wing leading edge concept

how the load path selection method in Section III may be used to restrict the number of parameters whilst still ensuring the optimization algorithm is able to investigate as much of the design space as possible.

Section V illustrates how the generalized optimization process may be applied to the compliant wing leading edge problem, which results in the generation of a suitable structure. Modifications made to this design to enable the construction of a physical demonstration model are described in Section VI, and suggestions are made for ways to extend the process for more complex problems in Section VII before conclusions are drawn.

\section{Optimization Problem Definition}

A generalised description of a structural topological and geometrical optimization problem is shown in Fig. 2; this is the starting point of the design process. ${ }^{5,6}$ It is necessary to define the terms in the figure. The

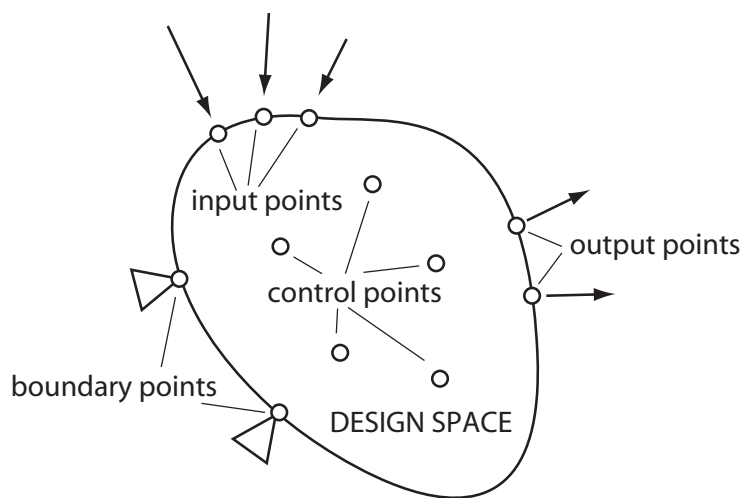

Figure 2. Generic problem definition for structural optimization

'design space' is the region of space within which the optimized structure must be constrained. For a twodimensional problem the design space may be defined by means of bounding curves, for three-dimensional problems, surfaces are used.

On the boundary of the design space lie the 'input points', 'output points', and 'boundary points' which are used to constrain the structures that are generated by the optimization algorithm further. These are respectively: points at which external loads and displacements are defined, points at which there is a desired output, and points at which the degree of connection to the ground may be defined. Within the design space are 'control points', which are used to define locations through which the structure must pass.

In addition, optimization objectives must be specified in terms of the minimization of desired functions, and further constraints, for example the maximum permissable stress, may be specified. The selection of 
these objectives and constraints is specific to the problem that is being considered.

\section{A. Starting Point}

Two primary techniques currently exist for providing a starting configuration for structural topology optimization. One is to fill the entire design space with material and permit the optimization algorithm to vary the material density. ${ }^{7}$ A variant of this technique maintains a constant material density but the material is either present or absent. In this approach, it is necessary to implement techniques that avoid unrealistic designs when the material is alternately present and absent over small distances - a phenomenon known as checkerboarding.

An advantage of this type of continuous optimization method is that the designer need not make any assumptions when setting up the problem. A considerable disadvantage is that the final design must be further subjected to a large amount of post-processing before it can be implemented as a physical structure. ${ }^{8}$ Such post-processing often requires a large amount of subjective decision on the part of the designer.

An alternative approach is to specify an initial structural network, of given topology, and to search for optimal structures whose topology is a subset of the original one. ${ }^{5,6}$ This means that the general structural form is already specified at the beginning of the design process and therefore minimal post-processing is required to realize a structure designed by this method. This approach also enables the inclusion of complex structural elements which would not be possible using the first approach. For example, the optimisation algorithm can vary the connection type between adjacent members of the lattice between rigid and perfectlyhinged. A structure containing localized hinges may be used as a pseudo-rigid-body model, which may then be realized as a compliant structure. ${ }^{1}$

There are, however, some disadvantages to this approach. For example, all generated structures are dependent on the initially-defined parent structure and therefore the quality of the solution is dependent on the suitability of this initial choice. In addition, if parent topologies with a large number of components are used - a requirement if the design space is not well understood - the number of parameters required to define the optimization can become prohibitively large.

Both of the above approaches have advantages but, because of the existing functionality of Samcef, it was decided to limit the optimization to lattice structures (consisting of beam elements) and to provide an initial topology as a starting point. Thus it was possible to utilize the parameterization facilities in Samcef ${ }^{15}$ and Boss-quattro to enable the optimization algorithm to generate subsets of the initial topology.

\section{B. Load Path Parameterization}

Having defined a parent structure consisting of a lattice of beam members, it is necessary to parameterize this structure to enable the optimization algorithm to remove elements in order to derive new topologies. An intuitive approach would be to assign binary parameters to the existence of each member where ' 1 ' corresponds to 'member exists' and ' 0 ' to 'member does not exist'. 5 This method of parameterization, however, leads to significant problems when it is combined with genetic search algorithms. Genetic Algorithms (GAs) are among the best tools for searching for optimal solutions when the design space is not well understood, as is usually the case for topological optimization. ${ }^{9,10}$ In addition, GAs have the advantage that they are less likely than gradient-based optimization algorithms to converge to local minima.

With the member parameterization scheme outlined above, GA iterations are very likely to generate disconnected structures over the course of the optimization; for example, there may be no load path between an input point and the rest of the structure. This will cause the optimization to fail.

A solution to this problem ${ }^{6}$ is to adopt an alternative parameterization scheme which assigns a binary parameter, not to individual members, but to sequences of members forming complete load paths. It can be shown that a connected structure will be generated provided there is at least one load path between each input and each output, each output and ground, and each input and ground. This is the topology parameterization scheme that is adopted in this paper. However, the automatic generation of load paths for a given parent lattice is by no means straightforward and is discussed in Section III.

The final topology determined by the GA is dependent only on the load path parameterization. However, the GA may be used simultaneously to determine the optimum geometry of the lattice structure which requires additional parameterization. This may include, among other things, the beam member cross-sections and the control point location within the design space. 


\section{Load-Path Determination}

The aim of this section is to present a systematic method for determining the load paths in a parent lattice, to enable a load path topology optimization.

It is first useful to illustrate the effects of increasing the complexity of the parent lattice on the number of possible load paths between two points. Figure 3 shows three lattice structures, each having the same unit cell. In all cases, we are interested in the number of possible load paths between the start node (shown shaded) and the end node (shown with a thick border). It is worthwhile to define exactly what is meant by a load path in this context. A load path is a direct route by which force may be transferred between two chosen nodes in a structure. A load path may therefore contain no closed loops and hence the same node must not appear more than once in any path definition.

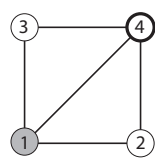

(a)

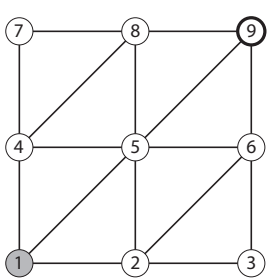

(b)

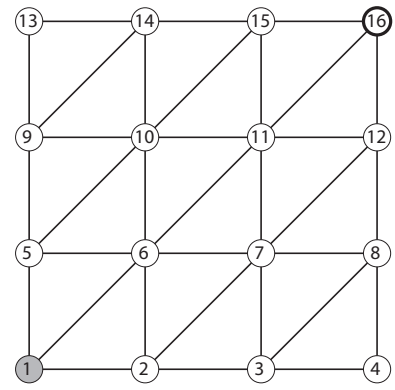

(c)

Figure 3. Lattices of increasing complexity

Figure 3(a) is the simplest lattice structure, consisting of 4 nodes and 5 members. It can readily be seen that there are three load paths between node 1 and node 4: [1 4 ], [ [ $\left.\begin{array}{lll}1 & 4\end{array}\right]$, and [ $\left.\begin{array}{lll}1 & 3 & 4\end{array}\right]$. At this scale of parent lattice, it makes no difference whether members or load paths are chosen to be parameterized. Figure 3(b) contains 9 nodes and 16 members. There can be shown to be 25 load paths between node 1 and node 9 . Increasing the number of members by approximately three times results in a greater than eight-fold increase in the number of parameters required to represent the lattice fully if load path parameterization is used. Finally, Figure 3(c) contains 16 nodes and 33 members. The number of load paths between node 1 and node 16 is 317. Thus, it is clear that, if load path representation is to be used, it is not practical to parameterize all the possible load paths for all but the simplest parent lattice structures.

\section{A. Load Path Selection}

A possible solution to the rapid increase in the number of load paths with lattice complexity is simply to limit the choice of parent structure to simple lattices, with only a few load paths. This is not a good solution, however, as limiting the complexity of the starting lattice limits the available topologies that may be generated as subsets of this initial structure. In order to minimise the effect of initial lattice selection (which is a subjective choice made by the designer) it is desirable that a more complex parent lattice be chosen to maximise the number of available sub-topologies.

There are two issues which must be addressed when a complex initial lattice is chosen. First, it is necessary to determine the number of load paths required to define the lattice, which may be very high. Second, it is necessary to restrict systematically the number of load paths that are parameterized in the analysis.

A possible restriction method is to limit the number of members that may be linked to form a load path. ${ }^{8}$ There are many lattices, however, for which this is not a suitable option. For example, in the lattice shown in Fig. 3(c) the shortest load path contains 3 members, there are then 12 load paths with 4 members, and 30 load paths with 5 members. Therefore, this technique provides the designer with limited means to set the level of parameterization of the design problem.

The solution proposed in this paper is to parameterize the $K$ shortest load paths, where $K$ is an integer value which may be defined by the designer. In the above example, if $K$ is set to 20, the parameterized 
load paths would include all the paths with 3 and 4 members, plus 7 load paths with 5 members. Which of the 5-member load paths are chosen for parameterization is determined by the order in which they are determined by the algorithm which is used to search for the load paths.

\section{B. $K$ Shortest Simple Paths (KSSP)}

In order to determine the $K$ shortest load paths, network analysis theory is used. The parent lattice is represented as an undirected graph $G=[E, N]$ in which $E$ is a set of all the edges in the graph (corresponding to the members in the parent lattice) and $N$ is the set of nodes $v_{i}$ linked by the edges in the graph. An undirected graph means that no distinction is made between the edges $\left[v_{n}, v_{m}\right]$ and $\left[v_{m}, v_{n}\right]$. This is the case here, as in a lattice structure this distinction is not needed. Additionally each edge has an associated cost $c$ representing, their relative weighting. In this case, however, there is no physical relevance of the weight as only the existence of a particular edge is of interest. Therefore they are equally weighted - in other words all edges have unit cost.

Having represented the parent lattice as a graph, the determination of the $K$ shortest load paths may be expressed as the well-known $K$ shortest path (KSP) problem in network analysis. The solution of the KSP problem was first attempted in 1959 in order to analyse the traffic flow in Detroit. ${ }^{11}$ However KSP algorithms permit paths with loops to be generated, but it has already been mentioned that in the present case the load paths should be loopless. In graph theory terminology this means that the paths must be 'simple'.

The solution of the $K$ Shortest Simple Path (KSSP) problem is significantly more complex than the solution of KSP. The best known solution to the KSSP problem is Yen's algorithm, for which a number of implementations have been proposed. ${ }^{12-14}$ By way of example, it is now shown how an initial iteration of Yen's KSSP algorithm for the lattice shown in Fig. 3(b) may be used to generate a list of load paths in increasing order of lengths (using the implementation described in [14]).

The graph representing this structure is shown in Fig. 4(a). It should be noted that despite the lengths of the lines of the sketch being different, each edge is assigned a unit cost (illustrated by the value in curly brackets). The load path start point is node 1 and the end point is node 9 . The first step of the algorithm is to determine a single shortest path using the well-known Dijkstra's algorithm.

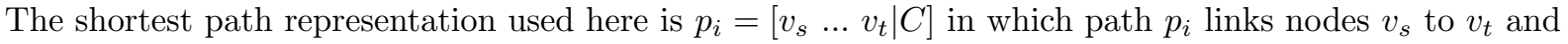
has a total weight $C$, equal to to the number of members in the load path. For the lattice in Fig. 4(a), Dijkstra's algorithm gives

$$
p_{1}=\left[\begin{array}{lll|l}
1 & 5 & 9 & 2
\end{array}\right]
$$

The next step is to remove the nodes and all emanating edges associated with the current (and any already-determined) shortest paths from the network, with the exception of the end node. The minimum tree rooted at the end node is then determined. This involves assigning a number to each node which represents the minimum distance from that node to the end node; this number is shown underlined in the figures. At this stage the network is as shown in Fig. 4(b).

The penultimate node of the shortest path is then reinserted into the network along with all its emanating edges except the edge present in the shortest path. The minimum tree rooted at the end node is then updated as shown in Fig. 4(c). At this point it is possible to determine some additional shortest paths which deviate from the current shortest path at node 5 . These paths are stored in a register, $[X]$, of candidate shortest paths

$$
[X]=\left[\begin{array}{llll|l}
1 & 5 & 6 & 9 & 3 \\
1 & 5 & 8 & 9 & 3
\end{array}\right]
$$

The edge emanating from node 5 and contained within the current shortest path is then reinserted in the network and the minimum tree rooted at the end node updated once more, as shown in Fig. 4(d).

The next node in the path (in this case the start node) is then reinserted into the network along with all its emanating edges, except the edge present in the shortest path, and the minimum tree updated in a manner similar to before. The resulting network is shown in Fig. 4(e). This enables additional candidate 


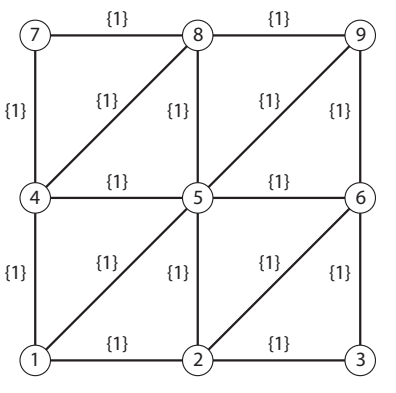

(a)

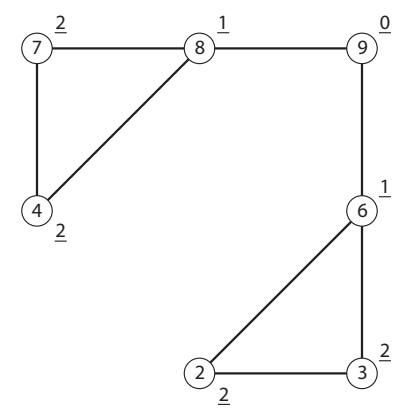

(b)

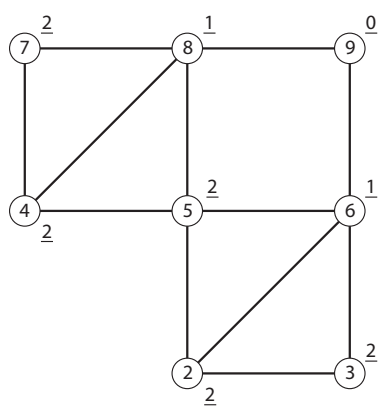

(c)

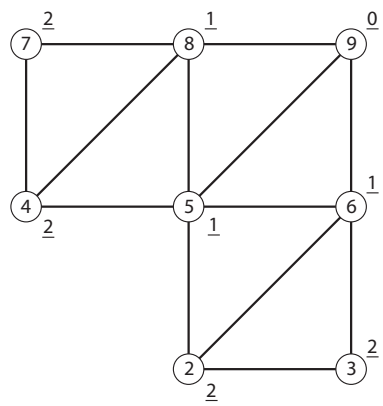

(d)

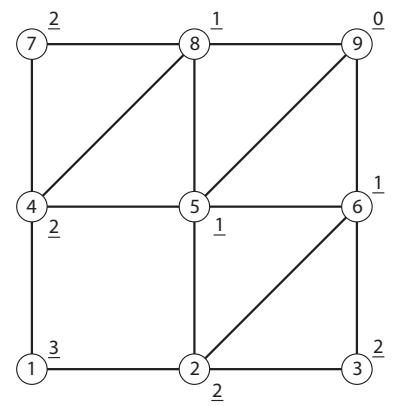

(e)

Figure 4. Illustration of a single iteration of Yen's Algorithm

paths to be placed in $[X]$ which deviate from the current shortest path at node 1 as follows

$$
[X]=\left[\begin{array}{llll|l}
1 & 5 & 6 & 9 & 3 \\
1 & 5 & 8 & 9 & 3 \\
1 & 2 & 5 & 9 & 3 \\
1 & 2 & 6 & 9 & 3 \\
1 & 4 & 5 & 9 & 3 \\
1 & 4 & 8 & 9 & 3
\end{array}\right]
$$

At this point the next shortest path is selected from the array of candidate paths $[X]$ as the path with the smallest value of $C$. If however, many candidate paths have the same length (as may be seen in Eq. 3) then the first candidate path with the shortest length is selected and removed from $[X]$. In this case

$$
p_{2}=\left[\begin{array}{llll|l}
1 & 5 & 6 & 9 & 3
\end{array}\right]
$$

The process is continued until either $[X]$ is empty or the number of shortest paths found is $K$. It can be seen that this technique provides a systematic method for a designer to control precisely the level of parameterization of the parent lattice, and, perhaps equally important, to determine how many load paths are not included in an optimization and consequently how many potential topologies are being neglected.

\section{Feasible Structure Generation}

It is clearly desirable that the optimization process results in an optimum structure which not only satisfies the imposed constraints but is also feasible to construct. Ensuring structural feasibility is, however, often neglected in the implementation of lattice structure topology optimization.

In particular, if the GA is free to place every control point anywhere within a two-dimensional design space there is a strong likelihood of generating structures with members that cross over each other, and which are therefore not physically realizable. In this paper, a method to prevent member cross-over is proposed. 


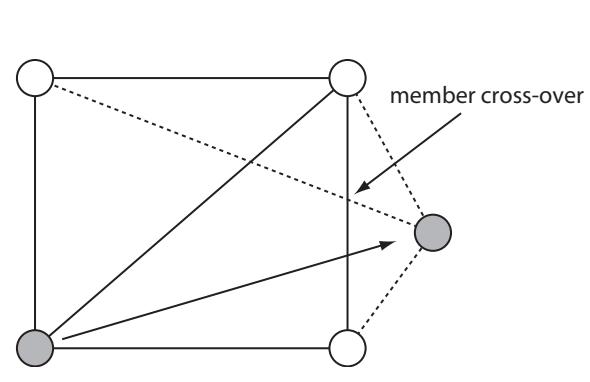

(a) Cross-over illustration

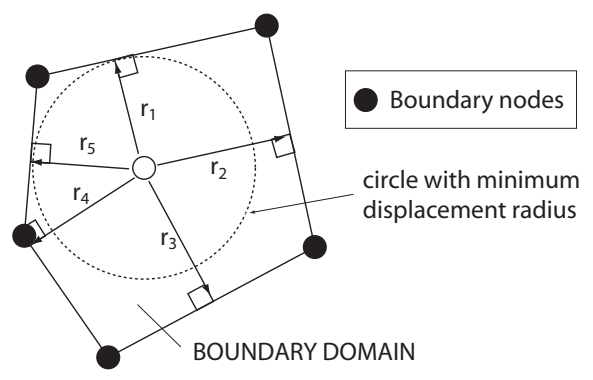

(b) Nodal boundary domain

Figure 5. Member cross-over avoidance

To a certain extent, the possibility of member cross-over can be reduced by careful choice of parent lattices that do not have crossing members. Using this criterion, all the lattices examined in Fig. 3 would be suitable candidates. However, if the control points may be placed anywhere within the design space such a lattice may still result in unfeasible structures. For example, if in Fig. 5(a) the shaded node is moved as shown by the optimization algorithm, while all other nodes remain in their original location, a member cross-over occurs.

A way of removing the possibility of member cross-over is to restrict the movement of control points within the design space. The technique proposed here is to assign all non-fixed nodes a series of boundary nodes which define a polygonal boundary domain, as shown in Fig. 5(b).

Each control point is assigned two geometric parameters which determine their displacement, in polar coordinates, from a known datum point. One geometry parameter determines the angle, and the other determines the fraction of a maximum permitted radius $r$. This radius is determined from the boundary nodes.

In Fig. 5(b) the control point is shown as a white circle. The boundary nodes, shown as black circles, are defined as the nodes to which there is a direct edge connection (not shown in the figure) from the control point. The boundary nodes form a closed polygon around the control point - the boundary domain. The minimum perpendicular distance $\left(r=\min \left(r_{1}, r_{2}, r_{3}, r_{4}, r_{5}\right)\right)$ from the control point to each of the boundary domain edges is defined as the maximum allowable radius of displacement that the control point may undergo. By restricting the geometric changes of the control point locations in this way, the possibility of member cross-over is removed.

\section{Optimization of Wing Leading Edge}

In this section, the optimization problem will be defined for the compliant leading edge rib introduced in Section I. It is envisaged that the wing will be used in an Unmanned Aerial Vehicle (UAV) and the dimensions are chosen accordingly. The wing profile is set as the NACA-2421 profile with a chord length $c$ of $1056 \mathrm{~mm}$ resulting in a leading edge of length $c / 4=264 \mathrm{~mm}$. The spanwise spacing between consecutive ribs is chosen to be $250 \mathrm{~mm}$, and the wing skin thickness is set at $1 \mathrm{~mm}$. An illustration of the optimization problem definition is provided in Fig. 6.

The finite element model used in the optimization is three-dimensional, and the skin is modelled using shell finite elements. A $5 \mathrm{~mm}$ wide and $0.5 \mathrm{~mm}$ thick interface is also provided between the compliant rib and the wing skin, and is also modelled using shell elements. Material properties were assumed to be those of isotropic High-Strength Aluminium and are listed in Table 1.

\begin{tabular}{||c|c||}
\hline Young's Modulus $\left(\mathrm{N} / \mathrm{mm}^{2}\right)$ & 72,000 \\
\hline Poisson's Ratio & 0.33 \\
\hline Yield Strength $\left(\mathrm{N} / \mathrm{mm}^{2}\right)$ & 395 \\
\hline
\end{tabular}

Table 1. High-Strength Aluminium material properties 


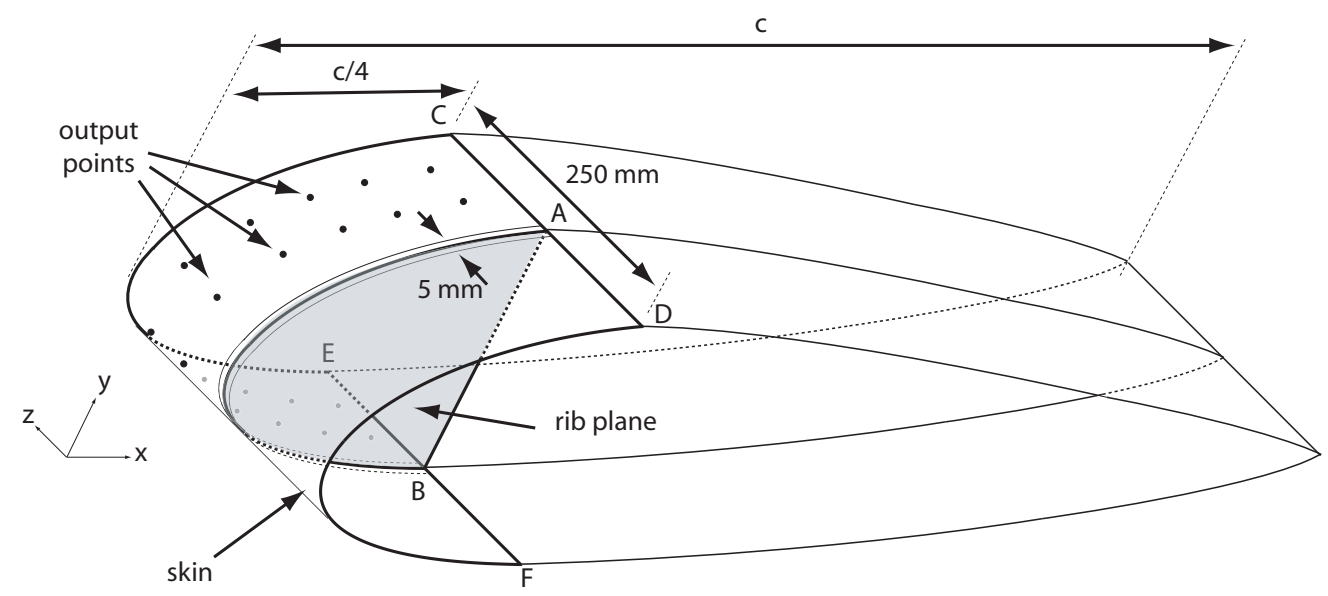

Figure 6. Adaptive leading edge optimization problem definition

\section{A. Optimization Objective Functions}

There are several optimization objectives that may be used, including minimizing the peak stress in the structure under actuation and minimizing the mass of the compliant rib. Also, the 'model complexity' can be minimized. This is a term which is related to minimizing the mass but is extended to provide a metric related to ease of construction.

If $i$ load paths $(p)$ are parameterized (with ' 1 ' corresponding to 'load path exists') then the model complexity may be defined as

$$
\text { model complexity }=\sum_{i} p_{i}
$$

However, if the connection type $(c)$ is also parameterized (with ' 1 ' corresponding to 'hinge connection') then the definition may be extended to

$$
\text { model complexity }=\sum_{i} p_{i}+\sum_{j} c_{j}
$$

in which $j$ is the total number of connection parameters. This is an advantageous optimization objective, as post-processing is required to implement rotational hinges as living hinges, which it is desirable to reduce. The form and number of objective functions is at the discretion of the designer. It has been observed, however, that the use of fewer objectives with more simple definitions substantially improves optimization convergence rates at the expense of increasing the post-processing requirements.

The primary objective function for the optimization, however, is the root-mean-square (rms) error of the actual deflection $d$ measured at the output point under actuation when compared to the desired deflection $\delta$. If there are $i$ output points the rms error objective function is defined as

$$
\text { rms error }=\sqrt{\frac{\sum_{i}\left(d_{i}-\delta_{i}\right)^{2}}{i}}
$$

For the compliant rib leading edge, the desired shape change is specified in terms of the vertical displacement of twenty points on the wing skin, arranged in two rows of ten (see the output points shown in Fig. 6). The reason for the two rows is to ensure there is no substantial variation of deflection in the spanwise direction. The desired shape change is equivalent to the vertical deflection that would result if the leading edge were rotated by $5^{\circ}$ downwards

\section{B. Loads and Boundary Conditions}

The FE model is fully-clamped at the interface between the leading edge and the rest of the wing. In Fig. 6 this corresponds to the lines CD and EF. The edges CE and DF are restrained against spanwise (z-axis) movement but are free to translate in the xy-plane. 
A single displacement actuation is applied to the compliant rib structure at along line AB. However, loading is also applied to the wing skin elements to represent the aerodynamic pressure loading that the wing is subjected to during flight. This pressure loading is calculated assuming a $260 \mathrm{kts}(134 \mathrm{~m} / \mathrm{s})$ air speed at sea level and a $5^{\circ}$ angle of attack. Inviscid flow is also assumed.

This is not an aeroelastic analysis - no account is taken of the change in pressure loading due to the change in wing profile under actuation - but it ensures that the compliant structure generated by the optimization algorithm accounts for the presence of an aerodynamic loading which, if not exact, will be of the correct order of magnitude.

\section{Compliant Rib Parameterization}

Although a three-dimensional model is used to represent the wing leading edge, the optimization problem is confined to the two-dimensional design space bounded by line $\mathrm{AB}$ and curve $\mathrm{AB}$ in Fig. 6 . The compliant rib is defined to be a lattice structure, modelled with beam elements having a fixed depth of $5 \mathrm{~mm}$. It is necessary to define a parent lattice as the basis of the optimization. The chosen lattice is shown in Fig. 7(a). It should be noted that the structure is free from member cross-over.

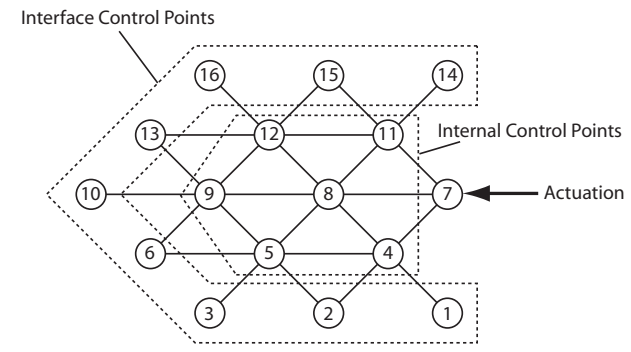

(a) Parent lattice graph

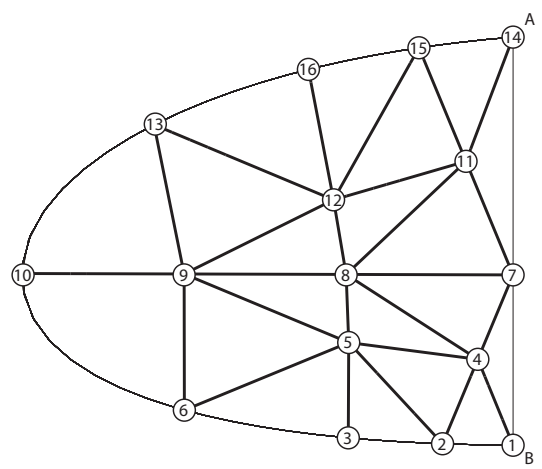

(b) Parent lattice realisation

Figure 7. Definition of parent lattice

The control points, which determine the geometry of the generated structures, are categorized as 'internal' and 'interface' control points. The former are free to move within the design space (subject to anti-cross-over constraints) whereas the latter are constrained to lie on the boundary, determined by curve AB in Fig. 7(b). The interface control points determine the locations where there is a connection between the rib and the wing skin.

For the purposes of defining a load path optimization, it is only necessary to ensure that there is at least one load path between the input (actuation) point and any one of the interface control points. All the remaining connections are ensured by the continuous presence of the wing skin. This is because the wing skin is fixed as described in Section B and the output points which are used to measure the shape change under actuation also lie on the skin. Therefore there is always a load path between the output points and ground, and from the interface control points to the output points.

In parameterizing the lattice in Fig. 7(a) 90 load paths were used -10 between node 7 and each of nodes $1,2,3,6,10,13,16,15$, and 14. An implementation of the idealized parent lattice onto the wing profile is shown in Fig. 7(b).

\section{Additional Parameterization}

Two sets of additional parameters are defined to vary the geometry and connections within the generated topologies.

The geometry parameters include the thickness of each beam (the depth is fixed), and the locations of: the input point, which is constrained to lie on line $\mathrm{AB}$; the internal control points, which are free to move within the design space; the interface control points, which are constrained to lie on curve $\mathrm{AB}$. In all cases the cross-over avoidance scheme described in Section IV is implemented. 
The connection parameter determines the connection type between adjacent members. These may be either rigid or perfect hinge connections. This enables the optimization to generate structures with both distributed and localized compliance.

\section{E. Optimization Settings}

The load path optimization was implemented using Samcef and Boss-quattro Full details may be found in [15], and only a brief summary is provided here.

A population size of 10 individuals is specified for the GA optimization. In each iteration there is a $90 \%$ probability of cross-over and a $1 \%$ probability of mutation. Individuals are selected for suitability by means of a tournament.

\section{F. Simplified Optimization Results}

An initial optimization was carried out using the minimization of the rms-error function (as defined in Eq. 7) and the model complexity (as defined in Eq. 5) as objective functions. All connections between adjacent members were defined as rigid, with the result that structures with only distributed compliance could be generated by the GA. The peak stress was not constrained.

An initial investigation was carried out to determine whether geometrically-nonlinear analysis was necessary. Linear analysis was found to be sufficient to model the deflections resulting from the actuation, and linear analysis was therefore used in the optimization. It is, however, necessary to carry out a geometricallynonlinear arc-length analysis of the final optimized structure to ensure that there is no buckling of the members under actuation.

Following 3000 iterations, the optimum structure shown in Fig. 8(a) was generated. A nonlinear analysis was then carried out to determine the shape change resulting from the displacement actuation. The result is shown in Fig. 8(b). It can be seen that there is no local buckling of the members. It should be noted that

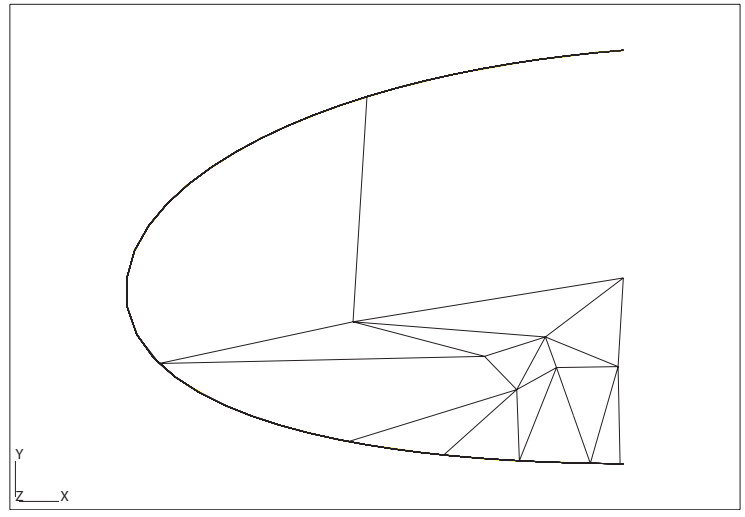

(a)

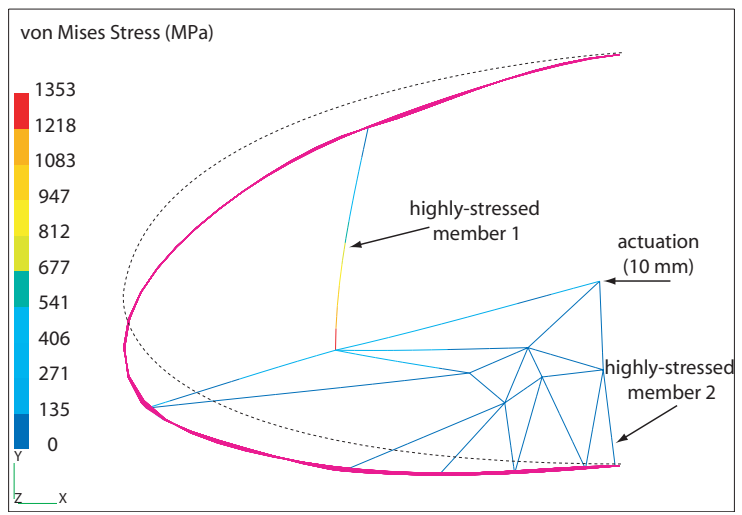

(b)

Figure 8. Optimized compliant leading edge

Fig. 8(b) is an end view of a three-dimensional structure. It can be seen that the deformed shape of the skin, modelled with shall elements, is not completely uniform, as there is a small amount of spanwise variation.

The rms error of the shape change is $0.83 \mathrm{~mm}$. It can be seen that the stress under actuation, including the effects of the aerodynamic pressure loading, is $1353 \mathrm{MPa}$. This is greater than the material yield stress, shown in Table 1 and must be reduced in a subsequent step.

\section{Refinement and Implementation}

This section illustrates the process of refinement and implementation of the optimized solution shown in Fig. 8(a) in order to realize a physical demonstration model of a single compliant rib.

The first stage is to remove the aerodynamic loading and the wing skin (leaving the connection strip defined in Section VII). This, in fact, has a limited effect on the peak stress, as the displacement actuation 
has a significantly greater effect on the stresses than the aerodynamic loading, and the highest stresses are in the rib members. The peak stresses are concentrated in the two members labelled as highly-stressed in Fig. 8(b). They are reduced in two different ways, as follows.

Member 1 is in tension, and high stresses in this member occur as a result of large bending moments at the ends. The solution is to replace the member connection type at each end with hinges which are implemented as living hinges. Member 2 is also subjected to high bending moments but it is in compression. Therefore, placing living hinges at the ends is not a good solution as they would reduce the resistance to buckling. Instead this member is simply removed as this is found to have only a minimal effect on the shape adopted by the rib under actuation. These modifications are shown in Fig. 9(a).

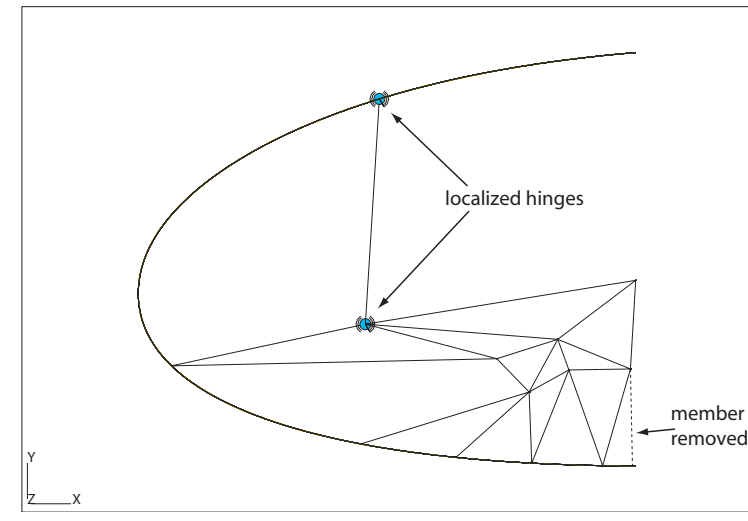

(a)

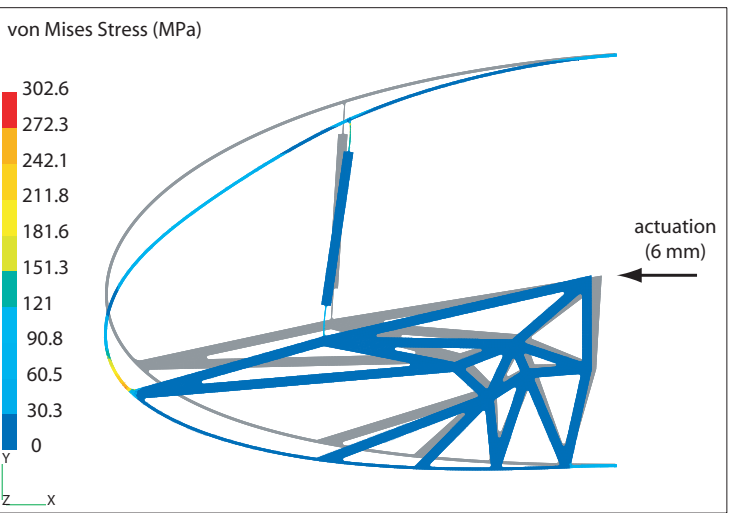

(b)

Figure 9. Manually-modified solution for the rib only

The hinges were realized as $0.5 \mathrm{~mm}$ thick living hinges. All corners were rounded to a radius of at least $1 \mathrm{~mm}$, to avoid stress concentrations. In order to confirm that the modified model behaved in the desired manner, a finite element model using solid elements was analysed. The shape change and von Mises stress of this modified model under actuation is shown in Fig. 9(b). The actuation displacement is reduced to 6 $\mathrm{mm}$ as the wing skin is not present to resist the shape change. It can be seen that the peak stress in the compliant rib is $303 \mathrm{~N} / \mathrm{mm}^{2}$ which provides a $30 \%$ margin against material yield.

\section{A. Physical Model}

A full-size demonstration model of the design shown in Fig. 9(b) was constructed. Figure 10(a) shows the model in its undeformed, unactuated configuration. Fig. 10(b) demonstrates the effect of a displacement actuation of $4.8 \mathrm{~mm}$ ( $80 \%$ of the actuation shown in Fig. 9(b)) applied by means of a screw. The undeformed shape is indicated by the black line. The model returns fully to its initial configuration when the actuation is removed.

\section{Optimization Extension}

The optimization and subsequent realization of a compliant wing rib, described in the previous sections, were simplified at various stages to provide an illustration of a complete design process from concept to a working demonstration model. However, it may be desirable to reduce the required degree of refinement of the final solution by the addition of further objective functions in the optimization problem, for example, one should consider minimizing the peak stress under actuation.

If the GA is permitted to vary the member end-connectivity, however, it is necessary to introduce further constraints to the optimization. This includes restricting the availability of end hinges to members that are in tension, and whose end-rotations would result in a peak strain in the living hinge that is well below the allowable limit for the chosen material.

Further restrictions should be considered to ensure that physically-feasible structures are generated. Prohibiting member cross-over is a necessary step. However, lattice structures whose members do not cross 


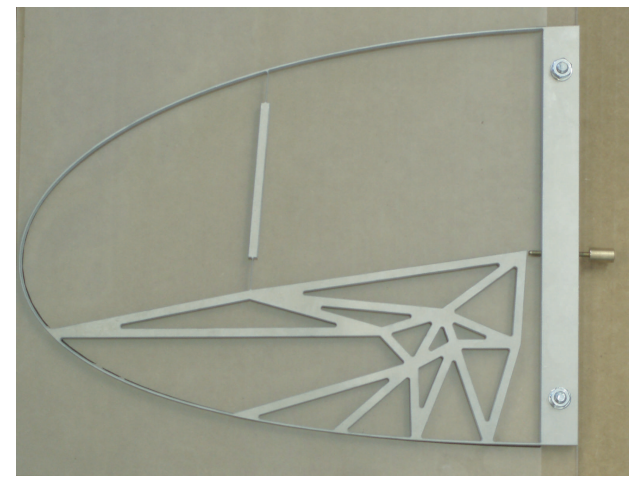

(a)

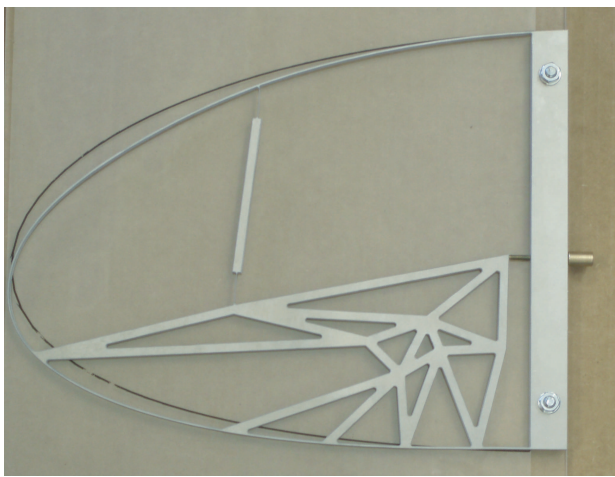

(b)

Figure 10. Demonstration model photographs

but are very close and nearly parallel should also be avoided. This could be accounted for by restricting the minimum angle between adjacent members, which would itself be a function of the member cross-sections.

Finally it should be noted that there are several benefits to implementing load path topology optimization techniques using commercial software. Primarily, the optimization may readily be extended for problems which require additional element types, such as membranes or solids, and additional loadings, for example, due to thermal effects.

Although linear analysis was found to be adequate for the compliant rib optimization, and nonlinear analysis was used only to verify the buckling resistance of the optimized structure, the use of commercial finite element software means that the facility to carry out optimizations for problems involving both geometric and material nonlinearity is already available.

\section{Conclusion}

The primary conclusion that may be drawn from the work presented in this paper is that a ' $K$ Shortest Simple Path' algorithm from network theory has been used to provide a designer with a substantial level of control over the level of parameterization adopted for a particular optimization problem. It has been shown that this technique allows ready assessment of the load paths required to parameterize complex parent lattice topologies, which are necessary if a design problem is not well understood.

It has been shown that a satisfactory topology may be generated for a compliant wing rib leading edge using 90 load path parameters, when several thousand would be required to fully parameterize the parent structure, thus leading to significant reduction in the complexity of the problem.

\section{Acknowledgments}

The authors would like to thank Ettore Baldassin, Aurelio Boscarino, and Giovani Carossa of AleniaAeronautica, Italy for their input into the design of the compliant wing. The assistance of Fréderic Cugnon and Philippe Andry of Samtech, Belgium with the use of Samcef and Boss-quattro is gratefully acknowledged. The work presented in this paper was funded under the Sixth Framework Programme of the European Commission.

\section{References}

\footnotetext{
${ }^{1}$ Howell, L., Compliant Mechanisms, John Wiley and Sons, 2001.

${ }^{2}$ Kota, S., Hetrick, J., Osborn, R., Paul, D., Pendleton, E., Flick, P., and Tilman, C., "Design and Application of Compliant Mechanisms for Morphing Aircraft Structures," Proceedings of SPIE, Vol. 5054, 2003, pp. 24-33.

3 "SAMCEF v12.0-03," Samtech Group, LIEGE science park, Rue des Chasseurs-Ardennais, 8B-4031 Liège (Angleur), BELGIUM.
} 
4 "BOSS-quattro v6.0-01," Samtech Group, LIEGE science park, Rue des Chasseurs-Ardennais, 8B-4031 Liège (Angleur), BELGIUM.

${ }^{5} \mathrm{Lu}$, K.-J. and Kota, S., "Design of Compliant Mechanisms for Morphing Structural Shapes," Journal of Intelligent Material Systems and Structures, Vol. 14, 2003, pp. 379-391.

${ }^{6} \mathrm{Lu}, \mathrm{K} .-\mathrm{J}$. and Kota, S., "An Effective Method of Synthesizing Compliant Adaptive Structures using Load Path Representation," Journal of Intelligent Material Systems and Structures, Vol. 16, 2004, pp. 307-317.

${ }^{7}$ Pedersen, C., Buhl, T., and Sigmund, O., "Topology Synthesis of Large-Displacement Compliant Mechanisms," Internation Journal for Numerical Methods in Engineering, Vol. 50, 2001, pp. 2683-2705.

${ }^{8} \mathrm{Lu}$, K.-J. and Kota, S., "Topology and Dimensional Synthesis of Compliant Mechanisms using Discrete Optimization," Journal of Mechanical Design, Vol. 128, 2006, pp. 1080-1091.

${ }^{9}$ Ponterosso, P. and Fox, D., "Going Organic: Using Evolution in Civils Design," Proceedings of ICE, Vol. 160, 2007, pp. $43-48$.

${ }^{10}$ Forrest, S., "Genetic Algorithms: Principles of Natural Selection applied to Computation," Science, Vol. 261, 1993, pp. $872-878$.

${ }^{11}$ Hoffman, W. and Pavley, R., "A Method for the solution of the Nth Best Path Problem," Journal of Association of Computing Machinery, Vol. 6, 1959, pp. 506-514.

${ }^{12}$ Clarke, S., Krikorian, A., and Rausen, J., "Computing the N Best Loopless Paths in a Network," Journal of Society of Industrial and Applied Mathematics, Vol. 11, 1963, pp. 1096-1102.

${ }^{13}$ Hadjiconstantinou, E. and Christofides, N., "An Efficient Implementation of an Algorithm for Finding K Shortest Simple Paths," Networks, Vol. 34, 1999, pp. 88-101.

${ }^{14}$ Martins, E. and Pascoal, M., "A new implementation of Yen's ranking loopless paths algorithm," Submitted, 2000. URL (http://www.mat.uc.pt/eqvm/cientificos/investigacao/Artigos/new yen.ps.gz).

${ }^{15}$ Santer, M. and Pellegrino, S., "Implementation of Load-Path Topology Optimisation in Samcef for the Generation of Compliant Structures," Proceedings of the 10th Samtech Users' Conference, 2007. 